\title{
Retreatment of children after surgery for acquired esotropia: reoperation versus botulinum injection
}

\author{
Jaime Tejedor, José M Rodríguez
}

\begin{abstract}
Aims-Two viable options were compared, reoperation and botulinum toxin injection, in the management of children who need retreatment after surgery for acquired esotropia.

Methods-47 strabismic children previously operated to correct an acquired esotropia were randomised to reoperation or botulinum toxin injection. Reoperation was undertaken in 24 of these patients and botulinum toxin injection in 23 of them. The percentage net change in distance deviation, the percentage of patients with successful motor outcome, detectable fusion, and stereopsis were compared 1 year after retreatment and at last visit (average follow up: 2.9 years in reoperation group, and 2.7 years in botulinum group). The motor success rate relative to time elapsed from initial surgery was evaluated.

Results-There was no significant difference in the motor and sensory outcomes between patients reoperated and treated with botulinum injection. The frequency of correction to within 8 prism dioptres of orthotropia was, respectively: $75 \%$ versus $69.56 \%$ at 1 year; $70.83 \%$ versus $60.86 \%$ at last visit. Botulinum injection could be more effective when performed within 3 months of initial surgery.

Conclusions-Botulinum injection is a rapid and safe procedure that may be as effective as reoperation in the management of children who need a secondary procedure after surgery for acquired esotropia.

(Br f Ophthalmol 1998;82:110-114)
\end{abstract}

27 August 1997

Table 1 Patient characteristics

\begin{tabular}{|c|c|c|}
\hline & $\begin{array}{l}\text { Reoperation } \\
\text { group }(n=24)\end{array}$ & $\begin{array}{l}\text { Botulinum toxin } \\
\text { group }(n=23)\end{array}$ \\
\hline & $\operatorname{Mean}(S D)^{*}$ & $\operatorname{Mean}(S D)^{\star}$ \\
\hline Age at onset of deviation (years) & $3.01(0.96)$ & $2.8(0.79)$ \\
\hline Age at initial surgery (years) & $3.56(1.53)$ & $3.29(1.28)$ \\
\hline Time between initial and secondary procedure (years) & $1.5(0.98)$ & $0.99(0.84)$ \\
\hline \multicolumn{3}{|l|}{ Angle before retreatment (prism dioptres) $\dagger$} \\
\hline Distance & $18.58(18.52)$ & $18.69(16.56)$ \\
\hline Near & $21.32(18.84)$ & $22.16(16.83)$ \\
\hline Spherical equivalent before retreatment (dioptres) & $2.21(1.3)$ & $1.98(1.07)$ \\
\hline $\begin{array}{l}\text { Visual acuity ratio before retreatment (amblyopic eye } \\
\text { when amblyopia exists/sound eye) }\end{array}$ & $0.88(0.11)$ & $0.89(0.09)$ \\
\hline Follow up after retreatment (years) & $2.9(0.81)$ & $2.7(0.42)$ \\
\hline
\end{tabular}

^No statistically significant difference (unpaired Student's $t$ test)

$\dagger$ Measured by the simultaneous prism and cover test.
Reoperations are frequently necessary in the management of acquired esotropia. Ocular alignment within 8 prism dioptres of orthotropia has been considered a successful motor outcome. Success rates of $50-75 \%$ with conventional surgery have been improved up to $90 \%$ after surgery for a prism adapted target angle in patients who respond with fusion to the preoperative wearing of prisms. ${ }^{12}$ Sensory fusion may be obtained in $34-69 \%$ of patients 6 months after surgery ${ }^{1}$ and in $39-75 \%$ at 1 year. $^{2}$ Therefore, between $10 \%$ and $50 \%$ of patients require a second procedure. Most of these patients have residual esotropic deviations whereas alignment overcorrections are uncommon. ${ }^{1-3}$ Some patients may need retreatment because they drift out of alignment with time after surgery.

In an attempt to overcome the necessity of a new operation ophthalmologists have made use of postoperative adjustment with botulinum toxin injection ${ }^{4-7}$ or surgery with adjustable sutures. ${ }^{8-11}$ The latter procedure requires cooperation and is not easy to perform until late childhood. Botulinum toxin injection has been reported to be effective in treating previously operated strabismic children, ${ }^{67}$ and even more effective in those with fusional potential. In this study we compared the efficacy of reoperation versus botulinum toxin injection as a retreatment procedure in children after surgery for comitant acquired esotropia.

\section{Methods}

\section{PATIENT SELECTION}

We selected patients for entry into the study who were less than 11 years of age, who underwent surgical correction of acquired esotropia and required a second procedure, in whom the initial surgery was carried out between 1989 and 1994. We included patients with documented onset of comitant esotropia after 1 year of life. The following candidates were excluded: patients with a distance to near difference of at least 10 prism dioptres, children with vertical deviations greater than 4 prism dioptres, alphabetic syndromes, nystagmus, profound amblyopia (four or more lines of difference in visual acuity), or those in whom accurate information concerning the onset of deviation or the amount and type of initial surgery were unavailable. Eligible patients were randomised to reoperation (24 patients) or 
Table 2 Treatment procedures

\begin{tabular}{lll}
\hline & Reoperation group $(n=24)$ & Botulinum toxin group $(n=23)$ \\
\hline Initial surgery: & & \\
recess-resect & 10 & 8 \\
$\quad$ initial misalignment & 6 & 3 \\
$\quad$ drifted out of alignment & 4 & 15 \\
bimedial recession & 14 & 9 \\
$\quad$ initial misalignment & 7 & 6 \\
$\quad$ drifted out of alignment & 7 & \\
Secondary procedure: & 10 (7 esotropes, 3 exotropes) & $3-10 \mathrm{U}$ of botulinum toxin A \\
recess-resect & 8 (esotropes) & injection in medial recti (22 \\
re-recession & 1 (exotrope) & 5 U of botulinum toxin A \\
bilateral recess & 5 (esotropes) & injection in lateral rectus $(1$ \\
bilateral resect & & exotrope) \\
& &
\end{tabular}

botulinum toxin injection ( 23 patients) to correct an angle greater than 10 prism dioptres. Table 1 summarises the characteristics of the two groups of patients.

EXAMINATION SCHEDULE AND PROCEDURES We performed refractions 30 to 45 minutes after instillation of $1 \%$ cyclopentolate hydrochloride. Hypermetropia greater than +2.00 dioptres was corrected with glasses. The angles of deviation were measured by the simultaneous prism and cover test and the prism and alternate cover test at 6 and 0.33 metres in the different gaze positions, or with the Krimsky method when the cover tests were impracticable. The Worth 4-dot, the Bagolini lenses, the Titmus, and the TNO test were used to evaluate the sensory state before and after the secondary treatment. Patients with amblyopia underwent occlusion therapy before retreatment.

Initial surgery was a bimedial recession or a recession-resection procedure not always performed by one of us, but patients included in the study had been treated with only one previous documented operation in which the amount and type of surgery were known. Reoperation was performed by one of us with careful dissection of muscles and removal of fibrotic tissue. When the initial surgery was a recess-resect we performed recess-resect of the other eye in the appropriate direction according to previously published surgical dosages. ${ }^{12}$ When a bilateral recession was the primary procedure we performed a bilateral resection of the lateral recti, ${ }^{12}$ unless the patient was never orthophoric after initial surgery or significant restriction was detected medially by forced duction at the time of reoperation. In these two circumstances we made minimal amounts of bilateral medial rectus re-recession following the recommendations of King et al, ${ }^{13}$ and the conjunctiva was always recessed. In the botuli-

Table 3 Motor outcome after secondary procedure

\begin{tabular}{|c|c|c|c|c|}
\hline & \multicolumn{2}{|c|}{ Reoperation group $(n=24)$} & \multicolumn{2}{|c|}{ Botulinum toxin group $(n=23)$} \\
\hline & At 1 year & At last visit & At 1 year & At last visit \\
\hline $\begin{array}{l}\% \text { Net change }{ }^{\star} \text { (mean) } \\
\text { (range) (Mann-Whitney test) } \\
\% \text { Successful outcomet } \\
\text { (range) }\left(\chi^{2} \text { test) }\right.\end{array}$ & $\begin{array}{l}81.31^{\mathrm{a}} \\
(25-100) \\
75^{\mathrm{c}} \\
(18 / 24)\end{array}$ & $\begin{array}{l}79.51^{\mathrm{b}} \\
(23-100) \\
70.83^{\mathrm{d}} \\
(17 / 24)\end{array}$ & $\begin{array}{l}73.45^{\mathrm{a}} \\
(20-100) \\
69.56^{\mathrm{c}} \\
(16 / 23)\end{array}$ & $\begin{array}{l}70.88^{\mathrm{b}} \\
(20-100) \\
60.86^{\mathrm{d}} \\
(14 / 23)\end{array}$ \\
\hline
\end{tabular}

$\star$ (Preoperative deviation - postoperative deviation/preoperative deviation $) \times 100 \%$. tDeviation at distance by the simultaneous prism and cover test of $\leqslant 8$ prism dioptres.

Comparisons between pairs a, b, c, and d did not show statistically significant difference. num toxin group, botulinum toxin type A (Botox, Allergan) was administered under topical anaesthesia alone $(0.5 \%$ proxymetacaine (proparacaine) hydrochloride) or in combination with mild general anaesthesia (ketamine intramuscularly or intravenously or nitrous oxide inhalation). We used the maximal dosages suggested by Scott et al. ${ }^{6}$ The toxin was injected into one or two (when more than $5 \mathrm{U}$ of total dose) recti muscles with electromyographic control. The treatment procedures are listed in Table 2.

The follow up varied from 20 to 38 months. For statistical comparison we used the net change of the distance deviation (6 metres), measured by the simultaneous prism and cover test, the motor, and sensory success rates. These variables were compared at 1 year after the second treatment and at last visit. The net change was obtained by the following formula: (preoperative deviation - postoperative deviation/ preoperative deviation) $\times 100 \%$. Successful motor alignment was defined as a distance deviation of 8 prism dioptres or less by the simultaneous prism and cover test. Fusion was detected by the Worth 4-dot at near, and the presence of stereopsis with the Titmus circles and TNO test (at least 480 seconds of arc).

The information recorded included the following: age of presentation and diagnosis of strabismus, refraction and best corrected visual acuity, age at first and second treatment (and time elapsed between the two), angle of deviation before retreatment, angle of deviation after retreatment, at 1 year, and at last visit, fusion ability and stereo perception after the secondary procedure, at 1 year, and at last visit, surgical procedures performed, and dose of botulinum toxin when pertinent.

\section{STATISTICAL METHODS}

The unpaired Student's $t$ test was used to evaluate differences between means of continuous data assumed to follow a normal distribution, and the Mann-Whitney's test for those that were not normally distributed. $\chi^{2}$ analysis or Fisher's exact test were used to compare percentages. Statistical significance was defined by the conventional cut off of 0.05 .

\section{Results}

We included in the study 47 patients. Twenty four patients were reoperated and 23 were treated with botulinum toxin injection. In the reoperation group, the second surgery was aimed at the correction of residual esotropia (undercorrection) in 20 of the 24 patients, while in four of them it was undertaken to achieve the correction of consecutive exotropia (overcorrection). In the botulinum toxin group, 22 of the 23 patients were esotropic (undercorrected) and only one of them was injected to correct a consecutive exotropia.

The mean age at initial surgery (3.56 (SD 1.53) versus 3.29 (1.28) years) and the average time elapsed between the first and the second procedure $(1.5(0.98)$ versus 0.99 (0.84) years) were not considered significantly different in the two groups. The angle of deviation at distance and near before the secondary correc- 
Table 4 Sensory outcome (\%) after secondary procedure

\begin{tabular}{llllll}
\hline & Fusion & & & \multicolumn{2}{l}{ Stereopsist } \\
\cline { 2 - 3 } \cline { 5 - 6 } \cline { 5 - 6 } & Reoperation group & Botulinum group & & Reoperation group & Botulinum group \\
\hline One year $\ddagger$ & $62.5(15 / 24)$ & $56.52(13 / 23)$ & & $54.16(13 / 24)$ & $47.82(11 / 23)$ \\
Last visit $\neq$ & $62.5(15 / 24)$ & $52.17(12 / 23)$ & & $50(12 / 24)$ & $43.47(10 / 23)$ \\
\hline
\end{tabular}

*Worth 4-dot at near.

†Titmus circles and TNO test (at least 480 seconds of arc).

$\ddagger$ No statistically significant difference $\left(\chi^{2}\right.$ test).

Table 5 Timing of the secondary procedure and relative motor success *

\begin{tabular}{|c|c|c|c|}
\hline & \multirow[b]{2}{*}{$\begin{array}{l}\text { Time between the two } \\
\text { procedures (months) }\end{array}$} & \multicolumn{2}{|l|}{ No of patients } \\
\hline & & $\begin{array}{l}\text { Reoperation group } \\
(n=24)\end{array}$ & $\begin{array}{l}\text { Botulinum toxin } \\
\text { group }(n=23)\end{array}$ \\
\hline Initial misalignment & $0-6$ & 5 & 6 \\
\hline $\begin{array}{l}\text { (early failure) } \\
\% \text { successful }\end{array}$ & $6-13$ & 8 & 8 \\
\hline at 1 year† & & $84.61(11 / 13)$ & $78.57(11 / 14)$ \\
\hline at last visit† & & $76.92(10 / 13)$ & $78.57(11 / 14)$ \\
\hline Drifted out of alignment & $12-25$ & 7 & 7 \\
\hline (late failure) & $25-39$ & 4 & 2 \\
\hline $\begin{array}{l}\% \text { successful } \\
\text { at } 1 \text { yeart }\end{array}$ & & $63.63(7 / 11)$ & $55.55(5 / 9)$ \\
\hline at last visit $\ddagger$ & & $63.63(7 / 11)$ & $33.33(3 / 9)$ \\
\hline
\end{tabular}

$\star$ Distance deviation by the simultaneous prism and cover test $\leqslant 8$ prism dioptres.

†No statistically significant difference (Fisher's exact test).

$\ddagger$ Difference approaches significance $(\mathrm{p}=0.11)$.

tion procedure was similar in each of the treatment groups (18.58 (18.52) versus 18.69 (16.56) for distant angle in prism dioptres by the simultaneous prism and cover test). The mean refractive error and follow up were also similar in the reoperation and botulinum toxin group. Based on these data we regarded the two groups as homogeneous (unpaired $t$ test).

The motor outcomes obtained in this study are summarised in Table 3 . The mean percentage net change in the angle of deviation at distance after 1 year of follow up produced in the reoperated patients $(81.31 \%)$ was greater than that produced in the botulinum toxin group $(73.45 \%)$, but a statistically significant difference in the percentage net changes was not found (Mann-Whitney test). These values did not essentially change over the follow up period.

The percentage of patients with successful motor outcome at 1 year in the reoperation group $(75 \%)$ and in the botulinum toxin group $(69.56 \%)$ were not considered different after statistical analysis ( $\chi^{2}$ test). The percentage of motor success declined over the follow up in the two treatment groups with no significant changes $(75 \%$ v $70.83 \%$, and $69.56 \%$ v $60.86 \%)$. The downward trend is apparently greater in the botulinum toxin group but with our data the two modalities of therapy are similar in this aspect at last examination $(70.83 \%$ v $60.86 \%)$.

Examination of the sensory state revealed similar percentages of patients with ability to

Table 6 Influence of early treatment in the botulinum toxin group (motor success ${ }^{\star}$ )

\begin{tabular}{|c|c|c|c|c|}
\hline & \multicolumn{2}{|c|}{3 Month limit } & \multicolumn{2}{|c|}{6 Month limit } \\
\hline & $<3$ months & $>3$ months & $<6$ months & $>6$ months \\
\hline$\%$ Successful & $100(5 / 5)$ & $61.11(11 / 18)$ & $83.33(5 / 6)$ & $64.7(11 / 17)$ \\
\hline
\end{tabular}

$\star$ Deviation at distance by the simultaneous prism and cover test of $\leqslant 8$ prism dioptres.

†No statistically significant difference (Fisher's exact test), but the difference before/after 3 months since primary procedure approaches significance $(\mathrm{p}=0.12)$. fusion on the Worth 4-dot and with detectable stereopsis in the two studied groups (see Table $4 ; \chi^{2}$ test). We suspected a trend to decline, between the 1 year examination and the last visit, in the number of patients with detectable stereopsis, to a greater extent than in the number of patients with fusion, but neither of them showed a demonstrable significant decay. Although this tendency seems to be greater in the botulinum group, in which some patients also lost their fusion ability between the 1 year and the last examination, significant differences were not found.

The interval of time between the two interventions and its influence on the motor success rates are shown in Table 5 . For patients not satisfactorily aligned after the first operation (early failures) there is no significant difference in the results obtained with reoperation and botulinum injection (Fisher's exact test). For patients who drifted out of alignment with time (late failures), we didn't find a difference in the success rates either (Fisher's exact test), but at the last visit the difference approached significance in this category.

In the botulinum toxin group, we compared the motor success rates in patients treated within 3 months of the primary procedure $(100 \%)$ and in those treated later $(61.11 \%)$. We also compared the success rates in patients treated within 6 months of the primary procedure $(83.33 \%)$ and after 6 months $(64.7 \%)$. As could be expected, the results were better in children injected within 6 months, and even more within 3 months of surgery, in whom the difference with those injected after 3 months approached significance, but the null hypothesis could not be rejected in any of the two comparisons (Fisher's exact test). These data are listed in Table 6 . Transient ptosis occurred in eight of the 23 patients injected with botulinum toxin $(34.78 \%)$ and transient vertical deviation was present in four of the 23 patients $(17.39 \%)$.

\section{Discussion}

The main conclusion of this study is that botulinum toxin injection may be as effective as reoperation in the retreatment of children with one earlier surgery procedure for acquired esotropia. The present findings indicate that the net change produced in deviation and the motor and sensory success rates are statistically similar in the two groups, although most of the figures obtained were apparently better in the reoperated patients. Both therapeutic modalities may be of equal value at least in patients with the reported average deviation and interval of time since the initial procedure, and after more than 2.5 years of average follow up. This may be true especially for patients with undercorrected residual esotropia, because most of the cases included in our analysis presented with this condition. A greater decline in the success rates or a differential behaviour of the two treatment groups after a longer follow up cannot be discarded.

Whether the samples studied are a fair representation of a theoretical population of patients unsuccessfully corrected after surgery 
for acquired esotropia or not is difficult to gauge. Our treatment groups were the result of selection criteria but the figures of undercorrected and overcorrected patients that resulted from this selection are comparable with previously reported incidences. ${ }^{1-3}$ It is not known how many of the patients included were prism responders (ability to fusion after prism adaptation) before initial surgery. Since the reported incidence of misalignment is greater in prism non-responders, this category of acquired esotropes may be well represented in the two groups.

The percentage of patients aligned within 8 or 10 prism dioptres after one strabismus operation for childhood or acquired esotropia is about $50-90 \%{ }^{1-3}{ }^{14}$ but little information is available relative to the percentages of success in reoperations. Helveston ${ }^{15}$ pointed out that after a reoperation, $33 \%$ of patients may need other surgery for realignment, which may be comparable with the percentage success in this study. King et $a l^{13}$ obtained $40.62 \%$ success rate after re-recession and $57.5 \%$ after bilateral lateral rectus resection, which compare less favourably with our results, but they include different categories of residual esotropes and with larger deviations than in the present study.

Many of the previous studies with botulinum toxin refer to adults with different types of strabismus and cause and nature of the deviation, usually not previously operated, so that the results obtained cannot easily be compared with our data. Studies including infants and children report satisfactory success rates even after long term follow up. ${ }^{5}$ Biglan et $a l^{4}$ injected 56 patients after surgery and obtained, for overcorrections (eight patients), $84 \%$ of corrected deviation with $87.5 \%$ of patients controlled and, for residual deviations (48 patients), $46 \%$ of corrected deviation and $41.6 \%$ of patients controlled, but these data include both esotropes and exotropes. They obtained better results in overcorrected patients than our global results, in which 22 of the 23 patients were residual deviations (only one overcorrection), whereas our outcomes were better than those they report in residual deviations. Magoon ${ }^{5}$ obtained, for esotropes, $85 \%$ correction in the amount of deviation and, for exotropes, $79-83 \%$, with a global successful motor outcome rate of $85 \%$. These percentages are better than our reported net change in the deviation and percentage of satisfactory alignment, but only two of the 85 patients were injected after incisional surgery. Our results agree with the study by Scott et $a l^{6}$ in the percentage of final success in undercorrected esotropia $(66 \%)$ and to a lesser degree in the percentage change they report in undercorrected esotropia (63\%).

Biglan et $a l^{4}$ found better outcomes with botulinum injection in overcorrection than in undercorrection after traditional muscle surgery and $\mathrm{McNeer}^{7}$ also considered it useful in the management of consecutive deviations. Scott et al ${ }^{6}$ reported higher percentage success only for overcorrected exotropia compared with undercorrected exotropia, but the results were better for undercorrected than for over- corrected esotropia. This may be explained by the greater concentration of singly innervated fibres in the medial rectus muscle, which will be more effectively paralysed by botulinum toxin. ${ }^{16}$ Moreover, in overcorrected esotropia (consecutive exotropia), a recessed medial rectus probably cannot shorten or retract any more after lateral rectus injection. Thus, undercorrected esotropes will respond better to treatment, although a previously recessed muscle requires deeper and scar tissue penetration to be injected. We injected only one patient with overcorrected esotropia, whose deviation changed from 16 to 10 prism dioptres of exotropia. Surgical consecutive esotropia (overcorrected exotropia) is much better corrected with botulinum injection than surgically undercorrected esotropia. Here, the profound efficacy of botulinum chemodenervation on a previously untouched medial rectus versus a surgically recessed medial rectus is seen, despite the disadvantage of shortening of a recessed versus a non-recessed lateral rectus. Finally, an exotropia is thought to be more successfully managed when it is the consequence of an operated esotropia than of a surgically undercorrected exotropia.

It is interesting to note that Scott et $a l^{6}$ only found a small difference in the success rates with botulinum toxin injection between previously operated and non-operated children, while surgically misaligned strabismus was not so effectively treated by toxin in adults, probably because of retained muscle elasticity in children. Although scar tissue poses difficulties for treatment with botulinum toxin in operated patients, the angles of deviation are usually lower, which enhances the probability of success with this injection. In contrast, the efficacy of surgery is probably lower in operated than in non-operated patients as a result of adhesive and scar tissue, and so it approaches the success rates of botulinum toxin injection in surgically treated cases. The incidence of side effects after botulinum toxin injection is greater than in previously reported non-operated patients, ${ }^{4-6}{ }^{17-19}$ not only because it requires deeper and more difficult needle penetration, but also because in many patients we injected higher doses of the toxin.

The time interval after previous surgery may be a limiting factor. The limitations with both modalities of therapy are probably reduced as the length of time since previously performed surgery is shortened. Among patients initially misaligned after the first procedure, both reoperation and botulinum injection worked well. As a rule these patients probably should not be reoperated until 2 months after surgery and many of them may be managed with less aggressive measures. None the less, it seems reasonable to treat unsuccessful cases with botulinum toxin injection even earlier because it is a less invasive technique that often does not require general anaesthesia or only a brief superficial anaesthesia, and it is presumably much more effective when scar tissue and adhesions are not yet so restrictive. This procedure would increase or reverse the effect produced by surgery. Although we could not 
demonstrate benefit from early versus late postoperative treatment with botulinum toxin, the lack of statistically significant difference is probably a consequence of the insufficient number of patients injected within the first 3 or 6 months. The difference in motor success between patients injected before and after 3 months since previous surgery approached significance. In some of the patients included in the study, the interval between the first procedure and retreatment is long because, as depicted in Table 2, they were initially successfully corrected but drifted out of alignment with time. In this category of patients the two types of treatment were equally effective, but at last visit the difference in motor success rates of the two therapeutic modalities approached significance. This indicates that botulinum injection might not be as useful in late failures (or patients drifted out of alignment) as it is in those not satisfactorily aligned immediately after the primary procedure, compared with reoperation.

Our data could suggest that there is a downward trend of success rates with time in the two treated groups, but the difference observed between 1 year and last examination rates is not significant. The motor and sensory stability are apparently lower after botulinum injection. This assumption is not confirmed by statistical analysis. Previous studies have shown permanence of alignment after long term follow up of children treated with botulinum toxin, some of them after earlier surgery. ${ }^{56}$

A potential criticism is that some of the patients selected may not be within the diagnostic limits of acquired esotropia, a common objection to studies of this entity. We applied selection criteria very similar to those of the prism adaptation study ${ }^{1}$ to maximise the possibility that the participant children had an acquired esotropia. Since the age at onset as reported by the parents is frequently unreliable ${ }^{20}$ we only included children in whom documentary photographs and medical records indicate that the age of presentation was older than 1 year.

Another limitation of the study is the relatively small size of the samples. If any difference exists between the two treatments in this clinical setting, it is easier to uncover this difference with larger samples. Nevertheless, at the 0.05 significance level, the power of the tests used in this study is about $90 \%$, which is the probability of rejecting the null hypothesis when it is false.

According to the present findings, the retreatment of children after surgery for acquired esotropia with botulinum toxin injection can be considered safe and reasonable, and if the results were disappointing we still have an opportunity to repeat the injection or to perform surgery. With this procedure many patients may avoid a second operation with a rapid and less invasive technique.

1 Prism Adaptation Study Research Group. Efficacy of prism adaptation in the surgical management of acquired esotropia. Arch Ophthalmol 1990;108:1248-56.

2 Repka MX, Connett JE, Scott WE, Prism Adaptation Study Repka MX, Connett JE, Scott WE, Prism Adaptation Study
Research Group. The one-year surgical outcome after prism adaptation for the management of acquired esprism adaptation for the management
otropia. Ophthalmology 1996;103:922-8.

3 Bartley GB, Dyer JA, Ilstrup DM. Characteristics of recession-resection and bimedial recession for childhood esotropia. Arch Ophthalmol 1985;103:190-5.

4 Biglan AW, Burnstine RA, Rogers GL, Saunders RA. Management of strabismus with botulinum A toxin. Ophthalmology 1989;96:935-43.

5 Magoon EH. Chemodenervation of strabismic children. A 2- to 5-year follow-up study compared with shorter followup. Ophthalmology 1989;96:931-4.

6 Scott AB, Magoon EH, Mc Neer KW, Stager DR. Botulinum treatment of childhood strabismus. Ophthalmology 1990;97:1434-8.

7 McNeer KW. An investigation of the clinical use of botulinum toxin $\mathrm{A}$ as a postoperative adjustment procedure in the therapy of strabismus. 7 Pediatr Ophthalmol Strabismus $1990 ; 27: 3-9$.

8 Scott WE, Martin-Casals A, Jackson OB. Adjustable sutures in strabismus surgery. $\mathcal{f}$ Pediatr Ophthalmol Strabismus 1977;14:71-5

9 Jampolsky A. Current techniques of adjustable strabismus surgery. Am f Ophthalmol 1979;88:406-18.

10 Wisnicki HJ, Repka MX, Guyton DL. Reoperation rate in adjustable strabismus surgery. F Pediatr Ophthalmol Strabismus 1988;25:112-4.

11 Carruthers JDA, Kennedy RA, Bagaric D. Botulinum vs adjustable suture surgery in the treatment of horizontal misalignment in adult patients lacking fusion. Arch Ophthalmol 1990;108:1432-5.

12 Guidelines for strabismus surgery. In: Basic and clinical science course. Pediatric ophthalmology and strabismus. Am science course. Pediatric ophthal

13 King RA, Calhoun JH, Nelson LB. Reoperations for esotropia. F Pediatr Ophthalmol Strabismus 1987;24:136-40. 14 Kittleman WT, Mazow ML. Reoperations in esotropia surgery. Ann Ophthalmol 1986;18:174-7.

15 Helveston EM. Reoperations in strabismus. Ophthalmology 1979;86:1379-88.

16 Spencer RF, McNeer KW. Botulinum toxin paralysis of adult monkey extraocular muscle. Structural alterations in orbital, singly innervated muscle fibers. Arch Ophthalmol 1987;105:1703-11.

17 Magoon EH. Botulinum toxin chemo-denervation for strabismus in infants and children. I Pediatr Ophthalmol Strabismus 1984;21:110-2.

18 Magoon E, Scott AB. Botulinum toxin chemo-denervation in infants and children: an alternative to incisional strabismus surgery. F Pediatr 1987;110:719-22

19 Lingua RW. Sequelae of botulinum toxin injection. $A m f$ Ophthalmol 1985;100:305-7.

20 Costenbader FD. Infantile esotropia. Trans Am Ophthalmol Soc 1961;59:397-429. 